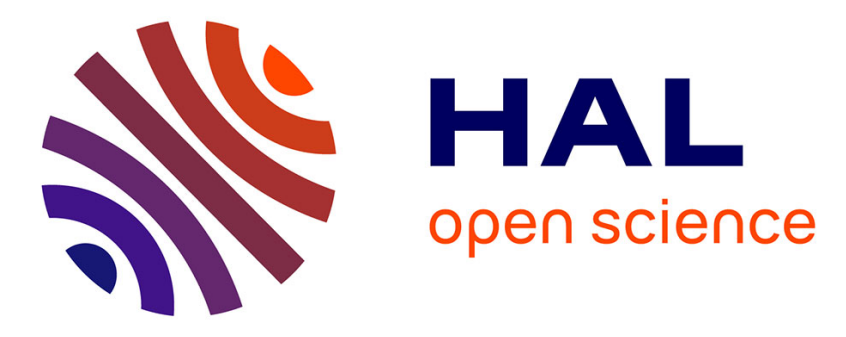

\title{
Comparaison des productions de chaleur en engraissement de porcs sur litière de sciure ou sur caillebotis intégral
}

Paulo Armando Victoria de Oliveira, Daniel Souloumiac, Paul Robin, Christophe Kermarrec

\section{To cite this version:}

Paulo Armando Victoria de Oliveira, Daniel Souloumiac, Paul Robin, Christophe Kermarrec. Comparaison des productions de chaleur en engraissement de porcs sur litière de sciure ou sur caillebotis intégral. Annales de zootechnie, 1999, 48 (2), pp.117-129. 10.1051/animres:19990204 . hal-00889786

\section{HAL Id: hal-00889786 https://hal.science/hal-00889786}

Submitted on 1 Jan 1999

HAL is a multi-disciplinary open access archive for the deposit and dissemination of scientific research documents, whether they are published or not. The documents may come from teaching and research institutions in France or abroad, or from public or private research centers.
L'archive ouverte pluridisciplinaire HAL, est destinée au dépôt et à la diffusion de documents scientifiques de niveau recherche, publiés ou non, émanant des établissements d'enseignement et de recherche français ou étrangers, des laboratoires publics ou privés. 


\title{
Article original
}

\section{Comparaison des productions de chaleur en engraissement de porcs sur litière de sciure ou sur caillebotis intégral}

\author{
Paulo Armando V. de Oliveira ${ }^{\text {a, b }}$, Daniel Souloumiac ${ }^{a}$, \\ Paul Robin ${ }^{\text {a* }}$, Christophe Kermarrec ${ }^{\text {a, }} \mathrm{c}$ \\ ${ }^{\text {a }}$ Bioclimatologie Inra, 65 rue de Saint-Brieuc, 35042 Rennes, France \\ ${ }^{b}$ EMBRAPA, Suínos e Aves, caixa postal 21, 89700 Concórdia, Brésil \\ ${ }^{c}$ ENSCR, CNGE, Avenue du Général-Leclerc, 35700 Rennes, France
}

(Reçu le 25 février 1998 ; 10 septembre 1998)

\begin{abstract}
Heat production by fattening pigs in deep-litter or conventional slatted floor systems. The insulation and ventilation requirements for livestock buildings depend on the amount of heat and water vapour produced by the animals. Our knowledge of heat production in slatted floor buildings must be adapted to the deep-litter system, in which there is early composting of the slurry. We isolated the effect of the husbandry system on heat and water vapour production using the same building, animals and external temperature. We compared the amount of heat and water vapour produced with the deep-litter system to that predicted by models, and to that of a conventional slatted floor system, used as a reference. Twelve pigs were fattened in two identical $14 \mathrm{~m}^{2}$ animal enclosures, with natural ventilation, and with external and internal temperatures kept constant $\left(13^{\circ} \mathrm{C}\right.$ and $\left.23^{\circ} \mathrm{C}\right)$. The total, sensible and latent heat productions were measured for the deep-litter housing during growing-finishing pigs and were equal to 302,127 and $176 \mathrm{~W}$, respectively. The litter produced a large amount of heat, which led to the evaporation of approximately $4 \mathrm{~kg}$ of water vapour $\cdot \mathrm{pig}^{-1} \cdot \mathrm{d}^{-1}$. This made it possible to maintain the internal temperature because there was a small but noticeable increase in sensible heat and significantly less heat loss through the floor. (@ Elsevier / Inra)
\end{abstract}

\section{pig / deep-litter system / heat production / water vapour production / livestock building}

Résumé - Les besoins d'isolation et de ventilation des bâtiments d'élevage dépendent des productions de chaleur et de vapeur d'eau des animaux. Les connaissances acquises pour l'élevage sur caillebotis doivent être adaptées à l'élevage sur litière qui réalise un compostage précoce des déjections. Nous avons déterminé l'effet du mode d'élevage sur les productions de chaleur et de vapeur d'eau en s'affranchissant des effets du bâtiment, des animaux ou du climat extérieur. Les productions de cha-

\footnotetext{
* Correspondance et tirés à part :
}

tél : (33) 0299285223 ; fax ; (33) 0299285430 ; e-mail ; probin@ roazhon.inra.fr 
leur et de vapeur d'eau d'un élevage sur litière ont été comparées aux résultats de modèles et aux productions d'un élevage sur caillebotis pris comme référence. Deux cellules d'élevage identiques de $14 \mathrm{~m}^{2}$, à ventilation statique, ont permis l'engraissement de 12 porcs de 30 à $100 \mathrm{~kg}$, avec des températures extérieure et intérieure maintenues constantes $\left(13^{\circ} \mathrm{C}\right.$ et $\left.23^{\circ} \mathrm{C}\right)$. Les productions de chaleur totale, sensible et latente ont été mesurées pour le couple animal-litière au cours de l'engraissement. Elles correspondent respectivement à $302 \mathrm{~W}, 127 \mathrm{~W}$ et $176 \mathrm{~W}$. La litière produit une quantité importante de chaleur qui participe à l'évaporation d'environ $4 \mathrm{~kg} \mathrm{d'eau} \mathrm{porc}{ }^{-1} j^{-1}$ et permet le maintien de la température interne par un faible apport de chaleur sensible et une baisse significative des pertes par le sol. (@ Elsevier / Inra)

porcs / élevage sur litière profonde / production de chaleur / production de vapeur d'eau / bâtiment d'élevage

\section{INTRODUCTION}

La technique d'élevage intensif des porcs sur litières profondes a été développée en Asie [18] afin d'apporter des solutions aux nuisances occasionnées par le lisier (odeurs et volume notamment). Elle permet en outre un traitement des effluents au travers d'un compostage précoce des déjections en profitant des conditions d'ambiance de l'élevage. En Europe, cette technique a été connue à la fin des années $80[1,28]$. L'adaptation des bâtiments destinés à ce mode d'élevage doit prendre en compte les productions de chaleur émises par le couple « animaux-litière ». Le calcul des besoins d'isolation et de ventilation des bâtiments fait intervenir d'une part le climat extérieur et sa variabilité saisonnière, et d'autre part la production de chaleur sensible et la production de vapeur d'eau à l'intérieur du bâtiment [7]. Pour calculer ces productions, les modèles utilisent la production de chaleur totale et son partage entre chaleur sensible et chaleur latente $[5,6]$; la production de chaleur sensible est la part de la chaleur totale à l'origine de l'élévation de température et la chaleur latente de l'évaporation d'eau. Cependant, ces modèles ne prennent en compte que les animaux dans le calcul de la production de chaleur totale.

La production de chaleur due spécifiquement à la litière est peu connue et varie probablement dans de fortes proportions selon la nature de la litière (paille, sciure ou copeau) et son humidité. L'importance de cette production de chaleur est mise en évidence par sa contribution à l'évaporation de l'eau des déjections $[16,23]$ et sa possibilité d'optimiser le processus de compostage de la litière.

Pour connaitre les modifications des productions de chaleur et de vapeur d'eau dues spécifiquement au mode d'élevage des animaux, il est nécessaire de conduire deux systèmes simultanément dans des conditions de bâtiment, de climat et d'animaux les plus proches possibles, de sorte que seul le mode de gestion des déjections diffère. Nous avons choisi comme référence un élevage sur caillebotis intégral durant une période d'engraissement de porcs (30 à $100 \mathrm{~kg}$ ). Pour la litière nous avons pris comme référence une litière ancienne (4 années), de préférence à une litière neuve. Seule la zone de déjections y était évacuée et remplacée par de la sciure neuve, brassée de temps en temps, comme cela se pratique habituellement en élevage sur litière profonde. La comparaison des modélisations existantes à nos mesures de flux sur caillebotis permet de valider la représentativité de notre expérimentation par rapport aux conditions pratiquées par la profession. Dans un second temps, la comparaison des mesures de flux de l'élevage sur litière profonde aux résultats 
des modèles permet de quantifier la modification des productions de chaleur et de vapeur d'eau à prendre en compte dans le calcul des caractéristiques d'isolation et de ventilation des bâtiments.

\section{MATÉRIEL ET MÉTHODES}

\subsection{Conduite de l'expérimentation}

L'expérimentation a été réalisée d'avril à juillet 1997 dans un local climatisé (12 mètres de long, 8,5 mètres de large et 4,5 mètres de haut) permettant la simulation de températures estivales ou hivernales.

Les enceintes d'élevage sont deux cellules identiques constituées de parois de $8 \mathrm{~cm}$ de polystyrène insérés entre deux contreplaqués de $2 \mathrm{~cm}$ d'épaisseur. L'isolation thermique diffère entre les deux cellules $\left(38,7 \mathrm{~W} \cdot{ }^{\circ} \mathrm{C}^{-1}\right.$ en cellule caillebotis et $26,5 \mathrm{~W} \cdot{ }^{\circ} \mathrm{C}^{-1}$ en cellule litière) la litière étant nettement plus isolante que l'ensemble caillebotis-fosse à lisier. Les cellules d'élevage sont ventilées statiquement et équipées de six convecteurs électriques ( $1410 \mathrm{~W}$ de puissance totale) utilisés en tension réduite pour permettre une émission d'air réchauffé, présentant un faible gradient thermique avec l'ambiance. La mise en fonctionnement de ces convecteurs est commandée par une centrale (AOIP type SA-120) qui enregistre les temps de mise sous tension des appareils pour chaque cellule indépendamment. La surface disponible des cellules d'élevage est de $14 \mathrm{~m}^{2}$. La surface utile par animal est celle couramment utilisée en pratique, soit $0,65 \mathrm{~m}^{2}$. porc ${ }^{-1}$ sur caillebotis et $1,10 \mathrm{~m}^{2} \cdot$ porc $^{-1}$ sur litière.

Les animaux utilisés pendant l'expérimentation ( 12 dans chaque cellule) sont des femelles de race Piétrain $\times$ Large White d'âge identique ( $\pm 2 \mathrm{j}$ de différence à la naissance), issues de la station de recherches porcines de Saint-Gilles (Inra). Les animaux ont été pesés en début d'expérience, au $46^{\mathrm{e}}$ et au $65^{\mathrm{e}} \mathrm{j}$ d'élevage, puis en fin d'expérimentation ( $90 \mathrm{j}$ ).

Les porcs sont nourris ad libitum durant toute la durée de l'expérimentation à l'aide d'un nourrisoupe (type Socobac) avec de la nourriture granulée. L'eau et l'aliment ont été pesés à l'aide d'une bascule Metler type TE $120 \mathrm{~J}$ (portée $120 \mathrm{~kg}$; résolution $\pm 0,05 \mathrm{~kg}$ ). La consommation d'eau des animaux a été mesurée de façon journalière par pesée de la quantité d'eau ajoutée dans les bacs et ajustée à un niveau défini. Cet ajout correspond en fait à la quantité d'eau consommée par les animaux lors de la journée précédente. De la même façon que pour l'abreuvement, la consommation d'aliment a été notée tous les jours par pesée de la quantité de granulés ajoutée pour compléter le nourri-soupe jusqu'à un niveau fixé. L'aliment était distribué tous les matins. L'aliment contenait en moyenne $3200 \mathrm{kcal} \cdot \mathrm{kg}^{-1} \mathrm{~d}$ 'énergie digestible (ED), $16 \%$ de protéines, $4 \%$ de matières grasses et $0,92 \%$ de lysine totale. La teneur en énergie métabolisable (EM) a été déduite du rapport $\mathrm{EM} / \mathrm{ED}=0,96[22]$.

L'expérimentation a été réalisée avec une température extérieure aux cellules de $12,8^{\circ} \mathrm{C}$ $( \pm 0,7 \mathrm{~K})$ et une humidité relative extérieure de $74 \%( \pm 7 \%)$. Les températures intérieures étaient respectivement de $23,1^{\circ} \mathrm{C}( \pm 0,7 \mathrm{~K})$ et $22,7{ }^{\circ} \mathrm{C}( \pm 0,6 \mathrm{~K})$ pour les cellules caillebotis et litière. L'humidité relative intérieure s'est établie en fonction du renouvellement d'air imposé par la constance des températures intérieure et extérieure. Elle a été de $66 \%( \pm 4 \%)$ en cellule caillebotis et de $75 \%( \pm 3 \%)$ en cellule litière.

La litière était constituée à base de sciure de bois. Elle était ancienne (4 années) et provenait d'un élevage de terrain. Elle avait déjà été utilisée pour l'engraissement d'environ 12 bandes de porcs. L'éleveur avait remplacé régulièrement la couche de litière dans la zone de déjection par une couche de sciure neuve. La litière a été pesée et analysée au début de l'expérimentation $(60,4 \%$ d'humidité pour une masse totale de $7111 \mathrm{~kg}$; $\mathrm{C} / \mathrm{N}=32 ; \mathrm{pH}=9 ; \mathrm{NTK}=4,7 \mathrm{~g} \cdot \mathrm{kg}^{-1}$ matière brute; $\mathrm{P}_{2} \mathrm{O}_{5}=5,5 \mathrm{~g} \cdot \mathrm{kg}^{-1}$ matière brute; $\mathrm{K}_{2} \mathrm{O}=7,1 \mathrm{~g} \cdot \mathrm{kg}^{-1}$ matière brute) et à la fin $(67,3 \%$ d'humidité pour une masse totale de $6632 \mathrm{~kg}$; $\mathrm{C} / \mathrm{N}=20 ; \mathrm{pH}=7,8 ; \mathrm{NTK}=5,9 \mathrm{~g} \cdot \mathrm{kg}^{-1}$ matière brute ; $\mathrm{P}_{2} \mathrm{O}_{5}=7,8 \mathrm{~g} \cdot \mathrm{kg}^{-1}$ matière brute ; $\mathrm{K}_{2} \mathrm{O}=11,4 \mathrm{~g} \cdot \mathrm{kg}^{-1}$ matière brute). Elle a été disposée en une couche de $70 \mathrm{~cm}$ et a été brassée manuellement tous les $15 \mathrm{j}$. La litière a été apportée en début d'engraissement, $75 \mathrm{~kg}$ de sciure ont été répartis sur la surface au $85^{\circ}$ jour d'élevage.

Le lisier a été pesé et analysé en fin d'expérimentation $(83,9 \%$ d'humidité pour une masse totale de $6632 \mathrm{~kg} ; \mathrm{pH}=8,2 ;$ NTK $=9,2 \mathrm{~g} \cdot \mathrm{kg}^{-1}$ matière brute; $\mathrm{P}_{2} \mathrm{O}_{5}=4,8 \mathrm{~g} \cdot \mathrm{kg}^{-1}$ matière brute ; $\mathrm{K}_{2} \mathrm{O}=16,4 \mathrm{~g} \cdot \mathrm{kg}^{-1}$ matière brute). 


\subsection{Mesures de température, d'humidité et de débit d'air}

Les mesures de températures sèche et humide de l'air ambiant interne des cellules et de l'air extérieur aux cellules sont réalisées par thermocouples type $\mathrm{K}$ reliés à une centrale d'acquisition type AOIP (SA-120). L'enregistrement de ces mesures est effectué automatiquement toutes les minutes et la moyenne toutes les heures permettant une précision de mesure de $\pm 0,1$ Kelvin. Les mesures de débit d'air sont effectuées manuellement sur les orifices calibrés d'extraction ( $10 \mathrm{~cm}$ de diamètre) de même diamètre que l'orifice de l'anémomètre. Les mesures ont été effectuées avec un anémomètre (type Air flow LCA-6 $000 ; 0,15$ à $12 \mathrm{~m} \cdot \mathrm{s}^{-1}$ ) une fois par jour et en continu sur un des orifices par une sonde anémométrique à fil chaud (TSI-Air Transducteur de vitesse d'air-modèle $8450 ; 0-2 \mathrm{~m} \cdot \mathrm{s}^{-1}$ ) relié à la centrale AOIP.

\subsection{Calcul des productions de chaleur mesurées}

Le schéma expérimental utilisé est présenté sur la figure $I$.

Les productions de chaleur observées sont déduites des mesures de débit d'air et des écarts de température et d'humidité entre l'intérieur et l'extérieur des cellules. Les débits d'air ont été calculés dans le cadre théorique du renouvellement d'air des bâtiments statiques par vent négligeable [27]. Nous avons préféré cette méthode à l'utilisation des observations brutes de vitesses d'air aux orifices de sortie d'air car (i) la mesure de vitesse d'air en continu n'était effectuée que sur un seul orifice et au centre de celui-ci (fil chaud) alors que dans la plage de vitesse utilisée $\left(0,2-0,8 \mathrm{~m} \cdot \mathrm{s}^{-1}\right)$ il existe une faible variabilité entre orifices ainsi que de faibles variations de vitesse dues uniquement à la convection naturelle (de l'ordre de $0,05 \mathrm{~m} \cdot \mathrm{s}^{-1}$ ), (ii) la mesure sur les 24 orifices de sortie par anémomètre à hélice
- Animaux phase de Croissance et Finition
Air de Sortie

Débit $\left(\mathrm{m}^{3} / \mathrm{h}\right)$

Tbs $\left({ }^{\circ} \mathrm{C}\right)$

Tbu ( $\left.{ }^{\circ} \mathrm{C}\right)$

$\left({ }^{\circ} \mathrm{C}\right)$ : température bulbe sec

Tbu $\left({ }^{\circ} \mathrm{C}\right)$ : température bulbe humide

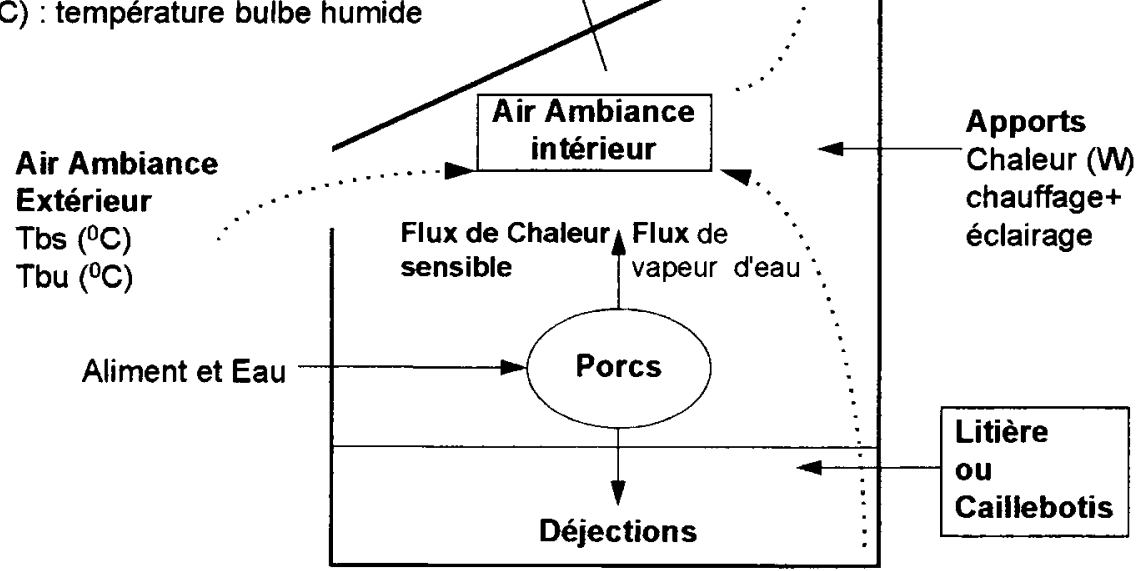

Figure 1. Bilan général de chaleur dans la cellule d'élevage de porcs. 
n'était effectuée que de temps en temps, (iii) les mesures de température et d'humidité étaient effectuées en continu sur l'air entrant et sortant et par ventilation des capteurs, (iv) la précision des mesures de température était nettement meilleure que celle des mesures de vitesse d'air, (v) la qualité des estimations de production de chaleur totale, sensible et latente dépendait fortement de la qualité des valeurs de débit d'air car les échanges thermiques convectifs ont largement dominé les échanges conductifs ou radiatif.

Dans ce cadre théorique le débit d'air est décrit par l'équation suivante [26].

$$
D=\alpha \cdot S \cdot \sqrt{g . Z \frac{\Delta T_{v}}{T_{v}}}
$$

où $\mathrm{D}$ est le débit d'air $\left(\mathrm{m}^{3} \cdot \mathrm{h}^{-1}\right), \alpha$ une constante d'efficacité des orifices (souvent de l'ordre de 0,7 ) dépendant des bordures et de la forme des orifices [27], $g$ l'accélération de la pesanteur $\left(9,81 \mathrm{~m} \cdot \mathrm{s}^{-2}\right), Z(\mathrm{~m})$ la différence de hauteur entre la section de sortie et la section d'entrée d'air, $S\left(\mathrm{~m}^{2}\right)$ est fonction des sections d'entrée et de sortie d'air, sa valeur est égale à la section d'entrée lorsque les deux sections sont identiques, $T_{v}$ (Kelvin) est la température virtuelle moyenne et $\Delta T_{v}$ l'écart de température virtuelle entre l'intérieur et l'extérieur de la cellule. La température virtuelle est déduite des mesures de température $\left(t\right.$ en ${ }^{\circ} \mathrm{C}$ ) et d'humidité spécifique ( $q$ en $\mathrm{kg}$ eau. $\mathrm{kg}^{-1}$ air sec) par l'équation suivante :

$$
T_{v}=(t+273,15) \cdot(1+0,61 \times q)
$$

L'équation (1) est transformée pour séparer le terme constant du terme variable du système qui dépend des climats interne et externe $\left(T_{v}\right)$ :

$$
D=(\alpha . S \cdot \sqrt{g \cdot Z}) \cdot \sqrt{\frac{\Delta T_{v}}{T_{v}}}
$$

Le terme constant a été estimé sur la base de l'ensemble des mesures de vitesse d'air pour chaque période où les sections des orifices d'entrée d'air sont restées constantes et après élimination des pas de temps perturbés par les ouvertures de porte ou le dépôt de poussière sur l'anémomètre. Ce terme moyen a ensuite été utilisé pour calculer le débit d'air à chaque pas de temps à partir des températures virtuelles.

La production de chaleur latente de l'ensemble animaux + litière est déduite de la quantité de vapeur d'eau évacuée par le débit d'air :

$$
\Phi_{l}=D \cdot \rho \cdot L_{v} \cdot \Delta q
$$

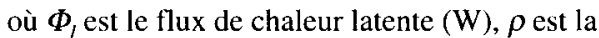
masse volumique de l'air humide ( $\mathrm{kg}$ air $\mathrm{sec} \cdot \mathrm{m}^{-3}$ d'air humide), $L_{v}$ est la chaleur latente de vaporisation de l'eau $\left(680,6 \mathrm{~W} \cdot \mathrm{h} \cdot \mathrm{kg}^{-1}\right.$ d'eau) et $\Delta q$ l'écart d'humidité spécifique entre l'air entrant et l'air sortant ( $\mathrm{kg}$ d'eau $\cdot \mathrm{kg}^{-1} \mathrm{~d}$ 'air sec).

Pour calculer la production de chaleur sensible de l'ensemble animaux + litière il faut déduire du flux évacué par le débit d'air $\left(\Phi_{s a}\right.$ en W) le flux de chaleur apporté par l'éclairage et le chauffage des cellules $\left(Q_{a}\right.$ en W, déduit des mesures de consommation d'énergie) et ajouter le flux de chaleur perdu à travers les parois $\left(P_{p}\right.$ en W) :

$$
\Phi_{s}=\Phi_{s a}-Q_{a}+P_{p}
$$

Le flux de chaleur sensible perdu par conduction à travers les parois $\left(P_{p}\right)$ est déduit de l'écart de température entre intérieur et extérieur $(\Delta T$ en Kelvin) et du coefficient de pertes conductives $\left(G_{p}\right.$ en W· $\left.\mathrm{K}^{-1}\right)$. Ce dernier a été mesuré en situation de régime permanent en apportant une quantité de chaleur connue à l'intérieur de chaque cellule :

$$
P_{p}=G_{p} \cdot \Delta T
$$

Le flux convectif est calculé de façon analogue au flux de chaleur latente:

$$
\Phi_{s a}=D \cdot \rho \cdot C_{p} \cdot \Delta T
$$

où $C_{\mathrm{p}}$ est la capacité thermique massique à pression constante de l'air humide. Celle de l'air sec est supposée constante et égale à $1004 \mathrm{~J} \cdot \mathrm{kg}^{-1}$ air $\mathrm{sec} \cdot \mathrm{K}^{-1}$. La capacité thermique massique à pression constante de l'air humide dépend pour sa part de l'humidité spécifique de l'air. Le facteur correctif $(1+0,84 . q$, d'après [24]) intervient pour moins de $1 \%$ autour de la valeur choisie

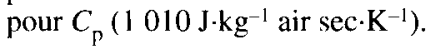

La chaleur totale $\left(\Phi_{t o t}\right.$ en W) produite par l'ensemble animaux + litiêre est égale à la somme des chaleurs sensible et latente :

$$
\Phi_{\text {tot }}=\Phi_{s}+\Phi_{l}
$$

Cette valeur observée est comparée aux modèles de la littérature.

\subsection{Calcul des productions de chaleur théoriques}

Il existe différents types de mesures et de modélisations du flux de chaleur d'origine méta- 
bolique émis par les porcs $[2,3,5,6,9-11,19$, $20,22]$. Le partage de ce flux total entre chaleur sensible et chaleur latente est estimé pour des conditions d'environnement variées où les animaux sont élevés sur sol de caillebotis intégral, béton, métallique ou sur paille $[2,3,5,6,9]$.

La production de chaleur minimale d'un porc à jeun, au repos et dans la zone de neutralité thermique où la température n'affecte pas son métabolisme, est désignée par la production métabolique basale $[8,22]$. Les études de Holmes et Close [12] ont déterminé la relation entre la masse corporelle des porcs et sa production de chaleur métabolique. Elle est estimée par l'équation suivante ; $Q t=k \cdot m^{n}$ où, $Q t=$ Flux de chaleur métabolique $(\mathrm{W}) ; m=$ masse corporelle des animaux $(\mathrm{kg}) ; \mathrm{k}=$ constante dépendant de la forme corporelle de l'animal ; $n=$ exposant variant entre 0,60 et 0,82 en fonction du type d'animal.

Nous avons privilégié les modélisations recommandées par la CIGR $[5,6]$ car elles résultent d'un accord entre scientifiques pour dimensionner des bâtiments. À titre de comparaison, nous avons aussi calculé la production de chaleur (totale, sensible et latente) par le modèle de Bruce [3] et la chaleur totale par le modèle de Noblet et al. [22] où nous avons utilisé l'équation :

$$
\text { Chaleur }=179,6 \times \mathrm{PV}^{0,60}+0,259 \times \mathrm{EM}
$$

Chaleur $=$ Production de chaleur $\left(\mathrm{kcal} \cdot \mathrm{j}^{-1}\right)$;

$\mathrm{EM}=$ Énergie métabolisable $\left(\mathrm{kcal} \cdot \mathrm{j}^{-1}\right)$;

$$
\mathrm{PV}=\text { Poids vif }(\mathrm{kg})
$$

La CIGR [5] a publié un ensemble d'équations représentatives des flux métaboliques émis par les porcs dans leur milieu d'élevage, en fonction de leur poids et de leur environnement climatique. La production de chaleur totale (CIGR, [5], $Q_{\text {to184 }}$ en W) pour les porcs à l'engrais à l'intérieur de la zone de neutralité thermique est estimée par :

$$
Q_{\text {tot84 }}=29 .(m+2)^{0,5}-40
$$

où $m$ est la masse du porc $(\mathrm{kg})$.

Dans la zone de neutralité thermique la production de chaleur totale est constante (CIGR, [5], $Q_{10192}$ en W). Son estimation pour les porcs est exprimée par l'équation suivante :

$$
Q_{t o t 92}=M E m+(1-k) \times(M E-M E m)
$$

où $M E m$ est l'énergie métabolisable utilisée pour l'entretien $\left(\mathrm{kJ} \cdot \mathrm{j}^{-1}\right), M E$ est l'énergie métabolisable ingérée $\left(\mathrm{kJ}^{\left.-\mathrm{j}^{-1}\right)} \mathrm{k}\right.$ est l'efficacité de l'utilisation de l'énergie métabolisable (sans dimension).
L'énergie métabolisable utilisée pour l'entretien est calculée par l'équation proposée par Close et Fowler (1983), cité par CIGR [6] dans les cas de porcs entre 20 et $120 \mathrm{~kg}$ :

$$
M E m=458 . m^{0.75}
$$

où $m$ est la masse du porc $(\mathrm{kg})$.

La CIGR [5, 6] recommande pour l'estimation de la production de chaleur sensible $\left(Q_{\mathrm{s} 92}\right.$ en W) l'équation suivante :

$Q_{s 92}=Q_{t o t 92} \cdot\left(0,8-1,85 \times 10^{-7} \times\left(t_{i}+10\right)^{4}\right)$

où $t_{\mathrm{i}}$ est la température interne de la cellule d'élevage $\left({ }^{\circ} \mathrm{C}\right)$. La production de chaleur latente $\left(Q_{192}\right.$ en W) se déduit simplement comme le complément à la chaleur sensible :

$$
Q_{i 92}=Q_{t o t 92}-Q_{s 92}
$$

Les valeurs théoriques ont été calculées pour les résultats zootechniques obtenus dans la cellule litière dans la mesure où notre objectif est d'améliorer les modélisations dans le cas des élevages sur litière, pour les besoins de conception de bâtiments. Les flux de chaleur observés sont d'abord confrontés aux estimations théoriques de la CIGR [5] où les quantités de chaleur produites par le métabolisme de l'animal ne dépendent que du poids et de la température de l'ambiance intérieure. Elles sont ensuite confrontées aux estimations de la CIGR [6] où les quantités de chaleur produites par le métabolisme de l'animal dépendent en outre de la transformation de l'énergie métabolisable contenue dans l'aliment ingéré.

\subsection{Traitement statistique des résultats}

Les variables mesurées et les flux de chaleur théoriques ont été traités par analyse de variance selon la procédure general linear model du SAS [25]. les comparaisons entre moyennes sont effectućes deux à deux par la méthode de Tukey. les résultats présentés aux tableaux sont les moyennes ajustées par la procédure LSMEANS du general linear GLM model [25].

\section{RÉSULTATS ET DISCUSSION}

\subsection{Performances des animaux}

La comparaison des résultats zootechniques des porcs élevés sur litière de sciure ou sur caillebotis intégral est présentée dans 
Tableau I. Comparaison des performances des animaux (moyenne et écart type de 12 porcs dans chaque système d'élevage) élevés sur litière ou sur caillebotis intégral.

\begin{tabular}{lcc}
\hline Résultats moyens & Caillebotis & Litière \\
\hline Poids d'entrée $(\mathrm{kg})$ & $29,8 \pm 1,23$ & $30,5 \pm 1,40$ \\
Poids de sortie $(\mathrm{kg})$ & $99,9 \pm 7,50$ & $102,3 \pm 7,96$ \\
Consommation totale d'aliment $(\mathrm{kg})$ & 190 & 192 \\
Consommation totale d'eau $(\mathrm{L})$ & $424^{\mathrm{a}}$ & $446^{\mathrm{h}}$ \\
Rapport eau / aliment $\left(\mathrm{L} \cdot \mathrm{kg}^{-1}\right)$ & 2,25 & 2,33 \\
Indice de consommation $\left(\mathrm{kg}^{\mathrm{l}} \mathrm{kg}^{-1}\right)$ & 2,71 & 2,67 \\
G.M.Q. global $\left(\mathrm{g} \cdot \mathrm{j}^{-1}\right)$ & 779 & 794 \\
Muscle F.O.M. $(\%)$ & $60,3 \pm 2,43$ & $60,9 \pm 1,77$
\end{tabular}

a. ${ }^{\mathrm{h}}$ Les valeurs affectées d'une lettre différente dans une même ligne différent statistiquement entre elles $(p<0,05)$.

le tableau I. En fin d'expérimentation le poids des animaux élevés sur litière est légèrement plus élevé, mais la différence n'est pas significative $(p<0,05)$. La consommation d'aliment, l'indice de consommation et la teneur en muscle des carcasses sont également identiques dans les deux traitements. La consommation d'eau tend à être plus élevée sur litière que sur caillebotis : $+22,7 \mathrm{~L} \cdot$ porc $^{-1}$ au total, soit un écart de $0,25 \mathrm{~L}$ de consommation journalière. Cette consommation peut être due soit à une activité plus intense des animaux dans la cellule litière que dans la cellule caillebotis, soit à un effet de la température de la litière sur le confort thermique des animaux dû à une température vécue plus chaude sur sol chaud que sur sol froid. Ces résultats zootechniques sont globalement en accord ceux obtenus par Hoy et al. [13], Bonazzi et Navarotto [1], Klooster et Grentink [16], Lesguillier et al. [17], Nicks et al. [21] et Kaufmann [15].

Les pourcentages de muscle à l'abattage, respectivement de 60,3 et 60,9 pour les animaux sur caillebotis et sur litière, ne diffèrent pas entre les traitements. Ces taux sont légèrement supérieurs aux résultats moyens des élevages en Bretagne [4, 14], vraisemblablement en raison de l'absence de mâles castrés dans notre étude.

L'homogénéité des résultats zootechniques permet de faire l'hypothèse d'homo- généité des flux métaboliques produits par les animaux dans les deux cellules.

\subsection{Production de chaleur mesurée et théorique}

Le tableau II présente les moyennes ajustées par la procédure LSMEANS du SAS [25], des productions de chaleur (W) mesurée et théorique, par porc, dans les cellules caillebotis et litière. Globalement, les valeurs observées sur caillebotis ne sont pas significativement $(p<0,05)$ différentes des valeurs théoriques. Ce résultat valide la représentativité de notre expérimentation par rapport aux conditions pratiquées par la profession. Pour la litière, les valeurs observées sont toutes significativement $(p<0,05)$ différentes des valeurs théoriques à l'exception de la chaleur sensible calculée par le modèle de Bruce [3].

\subsection{Production totale de chaleur}

La figure 2 présente l'évolution des productions de chaleur totale mesurée et théorique, dans les cellules litière et caillebotis, où $\Phi_{\text {tot }}$ représente le flux observé de chaleur totale émis soit par le couple animallitière dans la cellule litière, soit par le couple animal-fosse à lisier dans la cellule caillebotis. Les courbes $Q_{10184}$ et $Q_{10192}$ représen- 
Tableau II. Comparaison des moyennes ajustées des productions de chaleur (W) mesurées et théoriques par porc élevé sur litière profonde ou sur caillebotis intégral.

\begin{tabular}{lcccccccc}
\hline \multirow{2}{*}{ Chaleur } & \multicolumn{2}{c}{ Totale } & & \multicolumn{2}{c}{ Sensible } & & \multicolumn{2}{c}{ Latente } \\
\cline { 2 - 3 } \cline { 7 - 8 } Cellule & caillebotis & litière & & caillebotis & litière & caillebotis & litière \\
\hline$\Phi$ mesurée & $213^{\mathrm{aA}}$ & $302^{\mathrm{bA}}$ & $113^{\mathrm{aA}}$ & $127^{\mathrm{bA}}$ & $101^{\mathrm{aA}}$ & $176^{\mathrm{bA}}$ \\
CIGR-84 & $199^{\mathrm{aA}}$ & $200^{\mathrm{aB}}$ & & $110^{\mathrm{aA}}$ & $112^{\mathrm{aB}}$ & $89^{\mathrm{aA}}$ & $83^{\mathrm{aB}}$ \\
CIGR-92 & $186^{\mathrm{aA}}$ & $188^{\mathrm{aB}}$ & $102^{\mathrm{aA}}$ & $106^{\mathrm{aB}}$ & $88^{\mathrm{aA}}$ & $80^{\mathrm{aB}}$ \\
Bruce-81 & $175^{\mathrm{aA}}$ & $184^{\mathrm{aB}}$ & $117^{\mathrm{aA}}$ & $122^{\mathrm{aA}}$ & $85^{\mathrm{aA}}$ & $80^{\mathrm{aB}}$ \\
Noblet-89 & $202^{\mathrm{aA}}$ & $204^{\mathrm{aB}}$ & - & - & & - & - \\
\hline
\end{tabular}

ih Les valeurs affectées d'une lettre minuscule différente dans une même ligne montrent une différence significative entre systèmes d'élevage $(p<0,05)$. Als Les valeurs affectées d'une lettre majuscule différente dans une même colonne montrent une différence significative entre les valeurs observées et théoriques $(p<0,05)$.

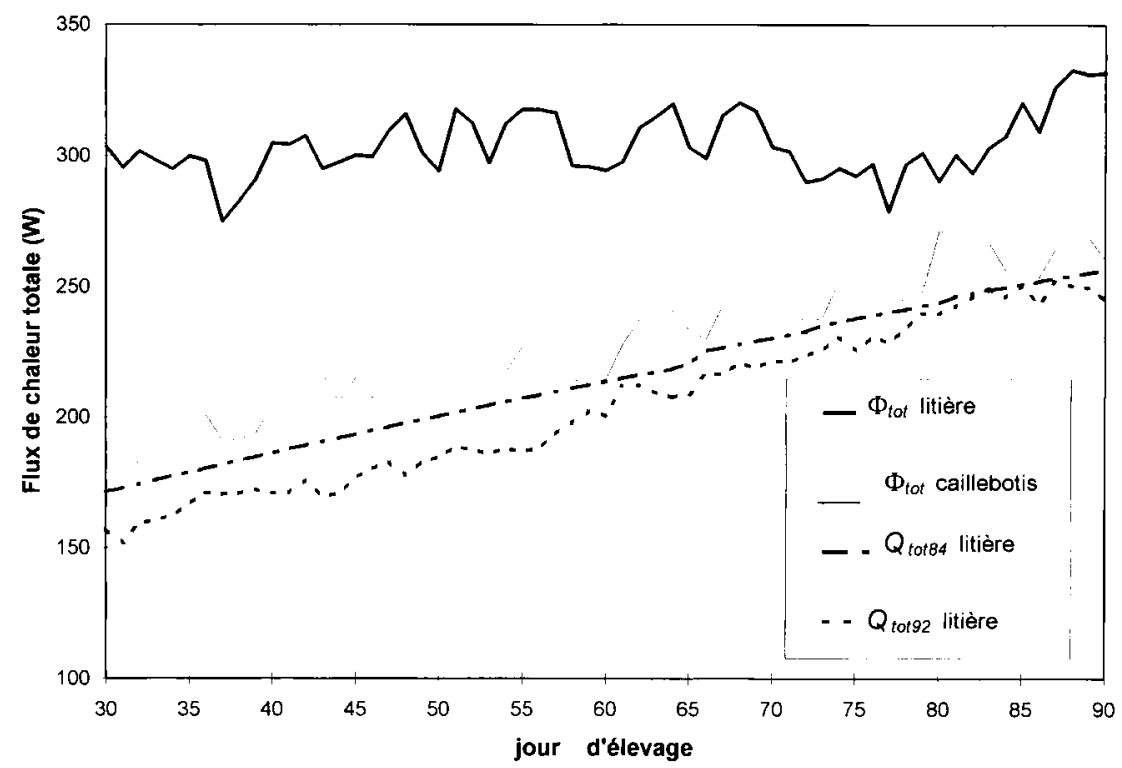

Figure 2. Évolution de la production de chaleur totale, par porc et par jour, observée et théorique dans des cellules d'élevage sur caillebotis intégral ou sur litière de sciure.

tent, respectivement, les estimations de la CIGR $[5,6]$ du flux de chaleur totale théorique produit par porc avec les données de poids des animaux et de consommation d'aliment de la cellule sur litière.

En système de caillebotis intégral, l'utilisation des équations de la CIGR $[5,6]$ pour l'estimation de la production de chaleur totale donne une bonne approximation de la contribution des porcs. Les petites variations observées sur la courbe $Q_{\text {tot92 }}$ sont liées aux variations de consommation d'aliment. En revanche ces équations sous-estiment largement la production de chaleur totale mesurée dans le système d'élevage sur litière. La plus forte sous-estimation observée lorsque les animaux sont jeunes (environ $60 \%$ du flux produit par les animaux entre 
30 et $50 \mathrm{j}$ ) à deux origines. D'une part les animaux produisent moins de chaleur $(170 \mathrm{~W}$ contre $250 \mathrm{~W}$ en fin de bande). D'autre part la litière est moins humide et produit plus de chaleur (plus de $100 \mathrm{~W}$ par animal en début de bande contre $60 \mathrm{~W}$ environ en fin de bande). Ces résultats confirment bien que pour estimer le flux de chaleur produit dans un système d'élevage de porc sur litière de sciure, il est indispensable d'ajouter le flux de chaleur produit par la litière dans les modélisations existant pour les animaux.

En attendant une modélisation plus fine de la production de chaleur par la litière en situation d'élevage, on peut utiliser la valeur présentée sur la figure 2, à savoir $302 \mathrm{~W}$ par animal, durant toute la phase d'engraissement pour le couple animal-litière. Cependant pour la conception d'un bâtiment, il est indispensable de connaître la répartition de ce flux entre chaleur sensible (principalement pour les besoins d'isolation) d'une part et chaleur latente d'autre part (principalement pour les besoins de ventilation).

\subsection{Chaleur sensible}

La figure 3 présente l'évolution des productions de chaleur sensible observées pour le couple animal-litière, pour la cellule caillebotis, ainsi que les valeurs déduites des modélisations de la CIGR $[5,6]$.

On constate que les quatre courbes sont en accord en fin de période d'engraissement (après $70 \mathrm{j}$ d'élevage). Les valeurs observées sont également en accord avec les recommandations de la DIN 18910 [7], 145 à $195 \mathrm{~W}$ de chaleur sensible, porc de 60 à $100 \mathrm{~kg}$ de poids vif. Les valeurs légèrement plus élevées de cette dernière référence peuvent être liées aux températures d'ambiance prises en compte $\left(12{ }^{\circ} \mathrm{C}\right.$ pris comme référence en fin d'engraissement par la DIN 18910 ) qui influencent le rapport chaleur sensible/chaleur totale.

En revanche les observations sur la cellule litière montrent qu'en début de période, l'usage des modélisations de la CIGR $[5,6]$ et DIN [7] conduirait à une très nette sousestimation de la production de chaleur sensible qui atteint $110 \mathrm{~W}$ à $40 \mathrm{~kg}$ de poids vif.

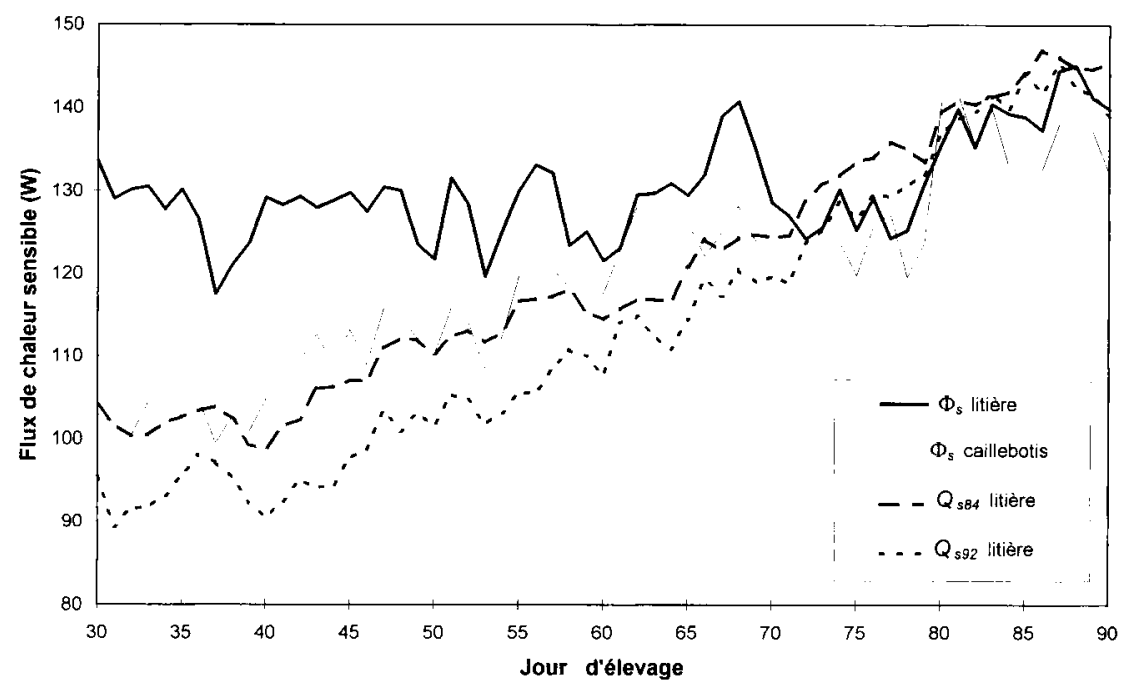

Figure 3. Évolution de la production de chaleur sensible, par porc et par jour, observée et théorique dans des cellules d'élevage sur caillebotis intégral ou sur litière de sciure. 
Cette sous-estimation diminue progressivement avec la croissance des animaux. Ceci s'explique probablement par l'augmentation progressive de l'humidité de la litière qui pourrait entraîner une moindre production de chaleur totale (moindre diffusion d'oxygène, figure 2). Pour les besoins pratiques de conception de bâtiment, on peut utiliser pour le couple " animal-litière », la valeur de $127 \mathrm{~W}$ de chaleur sensible par porc.

L'évolution du rapport entre les flux de chaleur sensible et de chaleur totale est présentée sur la figure 4. Les valeurs issues de la modélisation [6] sont proches des valeurs observées pour le caillebotis. En revanche les valeurs modélisées ne sont pas utilisables en élevage sur litière, dans la mesure où elles sont nettement plus faibles dans ce cas $(0,43$ en moyenne contre 0,56 pour les modélisations).

On constate une faible variation de ce rapport dans la cellule litière. La légère diminution jusqu'au $60^{\mathrm{e}}$ jour d'élevage peut s'expliquer par l'accumulation progressive d'eau dans la litière. La légère augmentation à partir du $65^{\mathrm{e}}$ jour d'élevage peut avoir deux origines. D'une part l'augmentation de la contribution des animaux dans la production de chaleur totale, d'autre part l'effet des brassages et de l'activité de fouissage des animaux qui relanceraient la production de chaleur de la litière. Cependant, ces évolutions restent faibles par rapport aux variations journalières et au besoin de précision pour la conception des bâtiments d'élevage.

\subsection{Vapeur d'eau}

Les productions de chaleur latente (vapeur d'eau) et eau dégagée par cellule $\left(\mathrm{kg} \cdot \mathrm{j}^{-1}\right)$ sont présentées sur la figure 5. Sur cette figure $\Phi_{\text {l litière }}(\mathrm{W})$ représente le flux de chaleur latente émis par le couple « animal-litière » dans la cellule litière et $\Phi_{1 \text { caillehotis }}(W)$ le flux de chaleur latente émis par le couple « animal-fosse à lisier » dans la cellule caillebotis intégral. $Q_{184}$ et $Q_{192}$ (W) représentent le flux de chaleur latente émis par l'animal dans la cellule litière selon les modélisations de la CIGR [5, 6].

On constate que les modélisations de la CIGR sont proches de la production de vapeur d'eau par la cellule caillebotis inté-

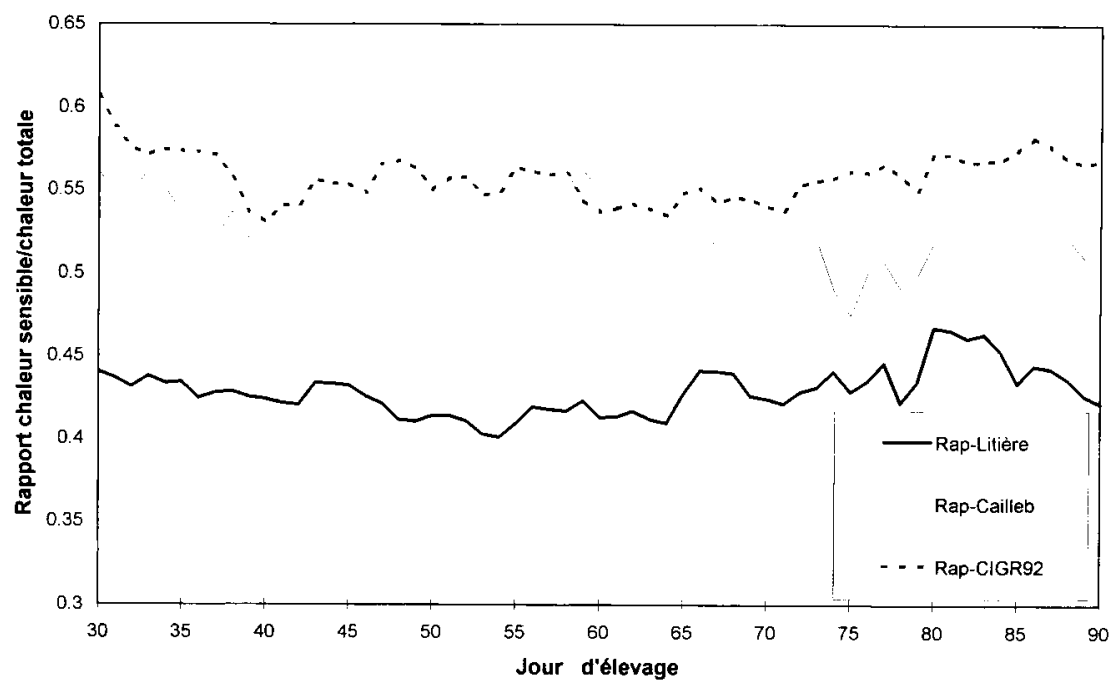

Figure 4. Évolution du rapport chaleur sensible/chaleur totale, par jour, observée et théorique dans des cellules d'élevage sur caillebotis intégral ou sur litière de sciure. 


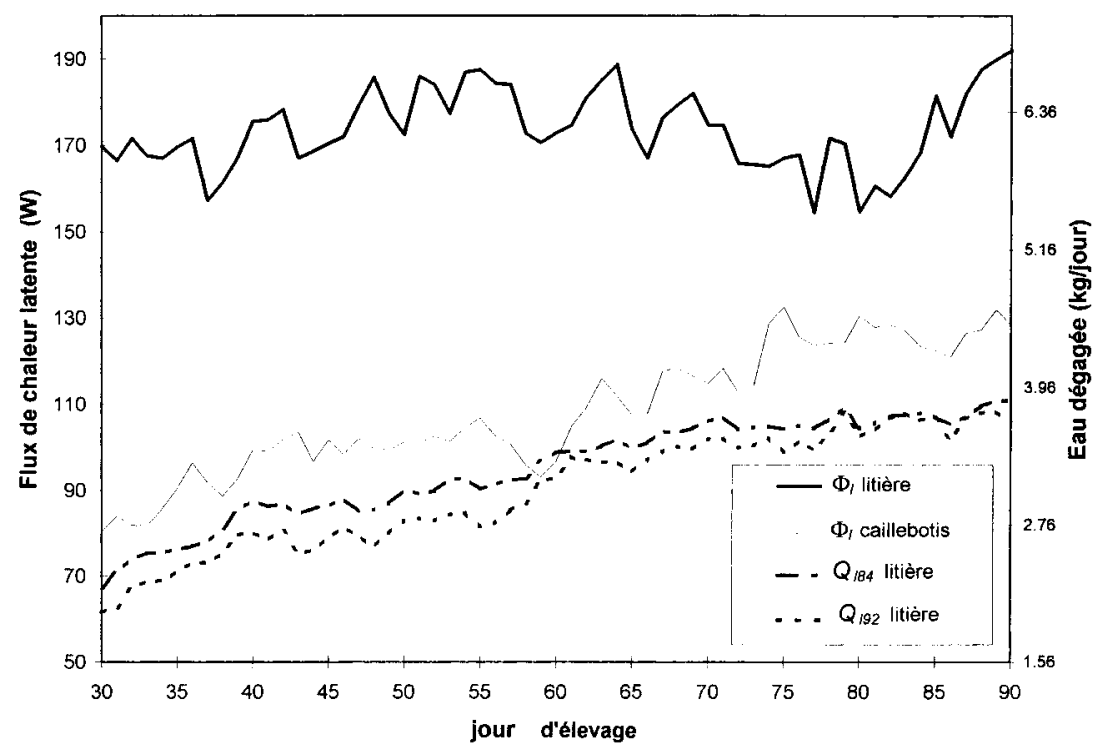

Figure 5. Évolution de la production de chaleur latente, par porc et par jour, observée et théorique dans des cellules d'élevage sur caillebotis intégral ou sur litière de sciure. Les quantités produites sont exprimées soit en Watt (axe de gauche) soit en $\mathrm{kg} \mathrm{d} \mathrm{d}^{\prime} \mathrm{eau} \cdot \mathrm{j}^{-1}$ (axe de droite), la conversion s'effectuant simplement à travers la chaleur latente de vaporisation de l'eau.

gral. En revanche la production de vapeur d'eau du couple animal-litière est largement supérieure à ces modélisations. De même les valeurs prises en compte dans la DIN 18910 [7] sont largement inférieures aux productions observées (DIN : 52 ou $92 \mathrm{~g}$ d'eau $\cdot \mathrm{h}^{-1}$ pour des porcs soit de $30 \mathrm{~kg}$ à $20{ }^{\circ} \mathrm{C}$, soit de $100 \mathrm{~kg}$ à $12^{\circ} \mathrm{C}$; valeur moyenne observée : $255 \mathrm{~g}$ d'eau $\cdot \mathrm{h}^{-1}$ ).

Par conséquent il est indispensable d'ajouter la production de vapeur d'eau par la litière pour estimer le besoin de ventilation des bâtiments sur litière. Ce besoin de ventilation est alors nettement supérieur à celui des bâtiments sur caillebotis intégral.

Les débits d'air mesurés ont varié entre 20 et $36 \mathrm{~m}^{3} \cdot \mathrm{h}^{-1}$. porc $^{-1}$ pour la cellule litière et entre 15 et $34 \mathrm{~m}^{3} \cdot \mathrm{h}^{-1}$. porc $^{-1}$ pour la cellule caillebotis. Les débits d'air évoluent en fonction du poids des animaux. La différence entre les deux cellules a varié de $5 \mathrm{~m}^{3} \cdot \mathrm{h}^{-1}$. porc ${ }^{-1}$ en début d'engraissement à $2 \mathrm{~m}^{3} \cdot \mathrm{h}^{-1}$. porc $^{-1}$ en fin d'expérimentation. Ces diffé- rences de renouvellement d'air se sont accompagnées néanmoins d'une ambiance légèrement plus humide dans la cellule litière.

La quantité totale d'eau sortant des cellules a été, sur l'ensemble de la période d'élevage, de $516 \mathrm{~L}$ pour la litière de sciure et de $278 \mathrm{~L}$ pour le caillebotis intégral, soit une différence de $238 \mathrm{~L}$ en faveur de la cellule litière (environ $4 \mathrm{~kg} \cdot \mathrm{j}^{-1}$ ). La mesure d'humidité de la litière au début et à la fin de l'expérimentation $(60,4 \%$ et $67,3 \%$ respectivement pour des poids de $7111 \mathrm{~kg}$ et $6632 \mathrm{~kg}$ ) indique qu'il y a eu une légère rétention d'eau dans la litière durant la période d'engraissement ( $14 \mathrm{~kg}$ eau porc $^{-1}$ ). Dans notre cas, cette eau résiduelle pouvait être évaporée au cours des deux semaines suivant le départ des animaux. On peut donc considérer que l'utilisation de la litière permet d'évaporer toute l'eau des déjections par rapport au système d'élevage sur caillebotis intégral. 


\section{CONCLUSION}

La représentativité des résultats expérimentaux présentés ici par rapport aux élevages réels a été vérifiée d'une part au travers des résultats zootechniques des animaux et d'autre part grâce au bon accord entre les modélisations de la CIGR $[5,6]$ et les résultats thermiques de l'élevage sur caillebotis intégral.

Nous pouvons imputer les différences observées entre les deux cellules au seul effet du système d'élevage (porc sur litière de sciure profonde) dans la mesure où les autres facteurs de variation ont été maintenus identiques entre les deux cellules : même enceinte d'élevage, mêmes climats extérieur et intérieur, mêmes résultats zootechniques, même consommation d'aliment et même rapport eau/aliment.

Dans ces conditions, nous avons observé que la production de chaleur totale du système porc-sur-litière est quasiment constante aux cours de l'engraissement ( $302 \mathrm{~W}$ par porc) contrairement au système d'élevage sur caillebotis où l'on passe d'environ $160 \mathrm{~W}$ à $260 \mathrm{~W}$ par porc. Il en résulte un besoin de ventilation et d'isolation moins dépendant du poids vif des animaux et un moindre besoin d'étanchéité du bâtiment en début d'engraissement. En ce qui concerne les productions de chaleur sensible et latente, celles-ci ont été mesurées de façon globale pour le couple animal-litière. Elles sont à peu près constantes au cours de l'engraissement et égales respectivement à $127 \mathrm{~W}$ et $176 \mathrm{~W}$ en moyenne. Dans la cellule caillebotis prise comme référence, la production de chaleur sensible a augmenté de $103 \mathrm{~W}$ à $136 \mathrm{~W}$ tandis que la production de chaleur latente a augmenté de $88 \mathrm{~W}$ à $127 \mathrm{~W}$.

Nous pourrions calculer la différence de vapeur d'eau produite par l'élevage sur litière et sur caillebotis, et faire l'hypothèse que la différence indiquée résulte de la production due spécifiquement à la litière. Cependant nous n'avons pu dissocier les flux issus des animaux et de la litière de façon à caractériser spécifiquement l'effet de cette dernière. En première approximation, il serait possible d'utiliser les modélisations de la CIGR pour estimer la contribution des animaux. Ces modélisations sont précises pour la production de chaleur totale mais restent incertaines pour la répartition entre les flux sensible et latent. En effet la répartition au niveau de l'animal est probablement influencée par la présence de la litière qui constitue une surface de couchage chaude et isolante par opposition au caillebotis. Par conséquent, on peut s'attendre à un besoin d'ambiance thermique plus froide en bâtiment litière qu'en caillebotis. Un travail supplémentaire reste à réaliser afin d'explorer la zone de neutralité thermique des porcs sur litière et de proposer des valeurs de température critique minimale pour ce type d'élevage. Ces valeurs sont nécessaires à la conception des bâtiments sur litière. Nous n'avons étudié ici qu'un seul type de litière et il serait intéressant d'avoir des mesures de production de chaleur pour des litières de paille, pour des litières de sciure moins épaisses et pour des ambiances d'élevage nettement plus froides.

\section{REMERCIEMENTS}

Nous remercions les partenaires du Comité Bretagne eau pure pour leur soutien financier, en particulier le Conseil régional de Bretagne, l'Agence de l'eau Loire-Bretagne et le Conseil général du Morbihan. Nous remercions Pierre Perrin (technicien de l'Inra) pour sa participation à la réalisation des dispositifs expérimentaux et la conduite des expérimentations. Nous remercions l'éleveur M. Le His et l'entreprise Guyomarc'h pour la fourniture de la litière et de l'aliment.

\section{RÉFÉRENCES}

(1) Bonazzi G.. Navarotto P.L, Littiera con trucioli di legno per suini in accrescimento-finissagio, L'Informatore Agrario, Verona, 34 (1992) 51-55.

121 Bruce J.M., Clark J.J., Models of heat production and critical temperature for growing pigs, Anim Prod. 28 (1979) 363-369. 
[3] Bruce J.M., Ventilation and temperature control criteria for pigs, Chapter IV-12, in: Clark J.A. (éd.), Environmental aspects of housing for animal production, Butterworths, London, 1981, p. 197-216.

14] Caugant A., Une stagnation par rapport à 1995 , Atout porc, mars (1997) 23-24.

[5] CIGR, Report of working group on climatisation of animal houses. Commission Internationale du Génie Rural, SFBIU, Aberdeen, 1984, $72 \mathrm{p}$.

[6] CIGR, $2^{\text {nd }}$ Report of working group on climatisation of animal houses, Commission Internationale du Génie Rural, SFBIU, Aberdeen, 1992, $147 \mathrm{p}$.

17] DIN 18910 , Wämeschutz geschlossener StälleWärmedämmung und Lüftung Planungs-und Berechnungsgrundlagen, Beuth Verlag GmbH, Burggrafenstrasse 6, 1000 Berlin 30, Mai 1992 , $17 \mathrm{p}$.

18] Esmay M.L., Principles of animal environment, AVI Publishing Co., Inc., Westport, CO, 1969.

[9] Feddes J.J.R., DeShazer J.A., Energetic responses of growing pigs to high cyclic and constant temperatures. Transactions of the ASAE (1988) 1203-1210.

[10] Feddes J.J.R., Parkhurst A.M., DeShazer J.A., Blecha A., Factor analysis of growing pigs' responses to high cyclic and constant temperatures, Transactions of the ASAE 5 (1996) 1847-1851.

[11] Henken A.M., Brandsma H.A., Van de Hel W., Verstegen N.W.A., Heat balance characteristics of limit-fed growing pigs of several breeds kept in groups at and below thermal neutrality, J. Anim. Sci. 69 (1991) 2434-2442.

[12] Holmes C.W, Close W.H., Nutrition and climatic environment, in : Haresign W., Swan, H., Lewis D. (éds.), Butterworths, London, (1977), pp. $51-74$.

[13] Hoy S., Kaczmare K.J., Ehser U., Fattening performance and animal health of keeping fattening pigs on a deep litter system with additives or on slatted floor, in: Voermans J.A.M. (éd.), Workshop on Deep-Litter System for Pig Farming, Research Institute for Pig Husbandry, Rosmalen, The Netherlands, 1992, pp. 57-76.

[14] ITP, Le porc par les chiffres, ITP, Paris, 1997, $40 \mathrm{p}$.

[15] Kaufmann R., Litière biomaîtrisée pour porcs à l'engrais, Journées de la recherche porcine en France 29 (1997) 311-318.

[16] Klooster V.C.E., Greutink G.J., Water evaporation in deep litter pig houses, in: Voermans
J.A.M. (éd.), Workshop on Deep-Litter System for Pig Farming, Research Institute for Pig Husbandry, Rosmalen, The Netherlands, 1992, pp. 124-134.

[17] Lesguillier F., Gouin R., Guiziou F., Orain B., Contribution au dossier environnemental par l'évaluation des rejets. Bilans des éléments azotés et minéraux des litières, Journées de la recherche porcine en France 27 (1995) 343-350.

[18] Lo C., Application and practice of the pig-on-litter system in Hong Kong, in: Voermans J.A.M. (éd.), Workshop on Deep-Litter System for Pig Farming, Research Institute for Pig Husbandry, Rosmalen, The Netherlands, 1992, pp. 11-25.

[19] Nienaber J.A., Maddy A.L., Temperature controlled multiple chamber indirect calorimeterdesign and operation, Transactions of the ASAE (1985) 555-560.

[20] Nienaber J.A., LeRoy G.H, Yen J.T., Thermal environment effects on growing-finishing swine, Transactions of the ASAE 6 (1987) 1772-1775.

[21] Nicks B., Désiron A., Canart B., Bilan environnemental et zootechnique de l'engraissement de quatre lots de porcs sur litière biomaîtrisée, Journées de la recherche porcine en France 27 (1995) 337-342.

[22] Noblet J., Fortune H., Dubois S., Henry Y., Nouvelles bases d'estimation des teneurs en énergie digestible, métabolisable et nette des aliments pour le porc, Inra, Paris, 1989.

[23] Oliveira P.A.V. de, Robin P., Kermarrec C., Souloumiac D., Dourmad J.Y., Comparaison de l'évaporation d'eau en élevage de porcs sur litière de sciure ou sur caillebotis intégral, Journées de la recherche porcine en France 30 (1998) 355-361.

[24] Queney P., Éléments de Météorologie, Masson, Paris, 1974.

[25] SAS, SAS user's guide, Statistical Analysis System Institute, Inc., Cary, NC, USA, 1990.

[26] Souloumiac D., Itier B., Prise en compte des phénomènes de chaleur latente dans la ventilation naturelle des bâtiments d'élevage, C.R. Acad. Sci. Série II (1989) 269-274.

[27] Souloumiac D., Étude des microclimats réalisables dans des enceintes énergétiquement autonomes soumises a des flux de chaleur d'origine métabolique, thèse Ina, Paris-Grignon, janvier $1995,170 \mathrm{p}$.

128] Voermans J.A.M., Preface, in: Voermans J.A.M. (éd.), Proceedings Workshop on Deep-Litter System for Pig Farming, Research Institute for Pig Husbandry, Rosmalen, The Netherlands, $1992,26-35$. 